\title{
OTIONOMICS
}

Revista de economía, empresa y sociedad

Dossier «Prevención de riesgos laborales: tendencias en tiempo de crisis»

\section{Fl impacto de los directivos sobre el estrés de los trabajadores}

\author{
Eva Rimbau-Gilabert \\ Profesora de la Universitat Oberta de Catalunya (UOC). \\ Estudios de Economía y Empresa
}

\begin{abstract}
RESUMEN El bienestar psicológico de los trabajadores deriva de un adecuado equilibrio entre, por un lado, los retos motivadores y los obstáculos a los que hacen frente y, por otro, los recursos laborales y personales con los que cuentan para superarlos. Los directivos o mandos están en una posición privilegiada para hacer posible este equilibrio, a través de su comportamiento diario, que se demuestra en su estilo directivo. Los estilos directivos pueden ordenarse según su impacto sobre el estrés laboral, de más negativo a más positivo: estilo abusivo, pasivo, transaccional y transformacional. El artículo explica las vías por las que los mandos pueden incidir en el bienestar psicosocial de los trabajadores y el papel concreto de cada uno de los estilos de liderazgo. Finalmente, concluye dando responsabilidad para desarrollar los estilos de liderazgo más positivos no solo a los mandos individuales, sino también a las empresas e instituciones y a las entidades formadoras.
\end{abstract}

PALABRAS CLAVE liderazgo; bienestar psicológico; dirección; estrés; salud laboral; liderazgo transformacional

\section{The impact of managers on employee stress}

\begin{abstract}
The psychological wellbeing of workers derives from a proper balance between, on the one hand, motivating challenges and obstacles faced and, on the other hand, job and personal resources to overcome them. Managers or supervisors are in a privileged position to make this balance possible, through their daily behavior shown in their leadership style. Leadership styles can be ordered according to their impact on job stress, from the most negative to the most positive style: abusive, passive, transactional and transformational. The article explains the ways in which managers can affect employees' psychosocial wellbeing and the specific role of each leadership style. Finally, it concludes by giving responsibility to develop more positive leadership styles not only to individual managers, but also to companies and institutions, as well as educational institutions.
\end{abstract}

KEYWORDS leadership; psychological wellbeing; management; stress; work health; transformational leadership 


\section{Introducción}

El trabajo tiene un gran impacto sobre el bienestar de las personas. Solo hay que pensar en la diferencia entre una tarde en casa después de un día de trabajo estresante, en comparación con un buen día de trabajo. Yendo a los datos, en Europa se ha calculado que entre un 50 y un $60 \%$ de los días de trabajo perdidos tienen algún vínculo con el estrés (Levi y Levi, 2000). Por tanto, es importante conocer cómo aparece el estrés en el trabajo, cómo se puede evitar que aparezca y qué factores pueden ayudar a los trabajadores a hacer frente a los inevitables factores estresores (como el elevado ritmo de trabajo) que a menudo son una parte inevitable del trabajo actual.

Las personas en posiciones de mando están en una situación privilegiada para evitar o disminuir el desarrollo de estrés y otros riesgos, y lograr el bienestar psicológico de sus colaboradores. ¿Cómo lo pueden hacer? Para aclararlo, primero explicaremos los mecanismos básicos del estrés laboral y, luego, analizaremos cuál es el impacto de la actuación de los directivos sobre el estrés de los trabajadores.

\section{1. ¿Cómo funciona el estrés laboral?}

Las características de los puestos de trabajo se pueden clasificar ${ }^{1}$, según su efecto sobre el estrés de los trabajadores, como «demandas» o como «recursos» (ver tabla 1 con ejemplos). Por un lado, las demandas laborales hacen referencia a aquellos aspectos físicos, psicológicos, organizativos o sociales del trabajo que requieren un esfuerzo sostenido y conllevan costes fisiológicos y psíquicos. Aunque no son necesariamente negativas, las demandas pueden convertirse en fuentes de estrés cuando hacerles frente requiere un elevado esfuerzo y el trabajador las asocia con costes altos, pero también cuando son demasiado reducidas. Por otro lado, los recursos laborales se refieren a los aspectos físicos, psicológicos, organizativos o sociales del trabajo que pueden (a) reducir las exigencias del trabajo y los costes fisiológicos y psicológicos asociados, (b) ser decisivos en la consecución de los objetivos del trabajo, o (c) estimular el crecimiento personal, el aprendizaje y el desarrollo. Además de los recursos laborales, los trabajadores también cuentan con recursos de tipo personal, que son aspectos de una persona que favorecen su resiliencia y se refieren al sentido que tiene el individuo sobre su propia capacidad para controlar y tener un impacto sobre su entorno (Xanthopoulou y otros, 2007).

\section{Tabla 1. Ejemplos de demandas y recursos con impacto sobre el estrés de los trabajadores (se} incluyen definiciones de algunos términos)

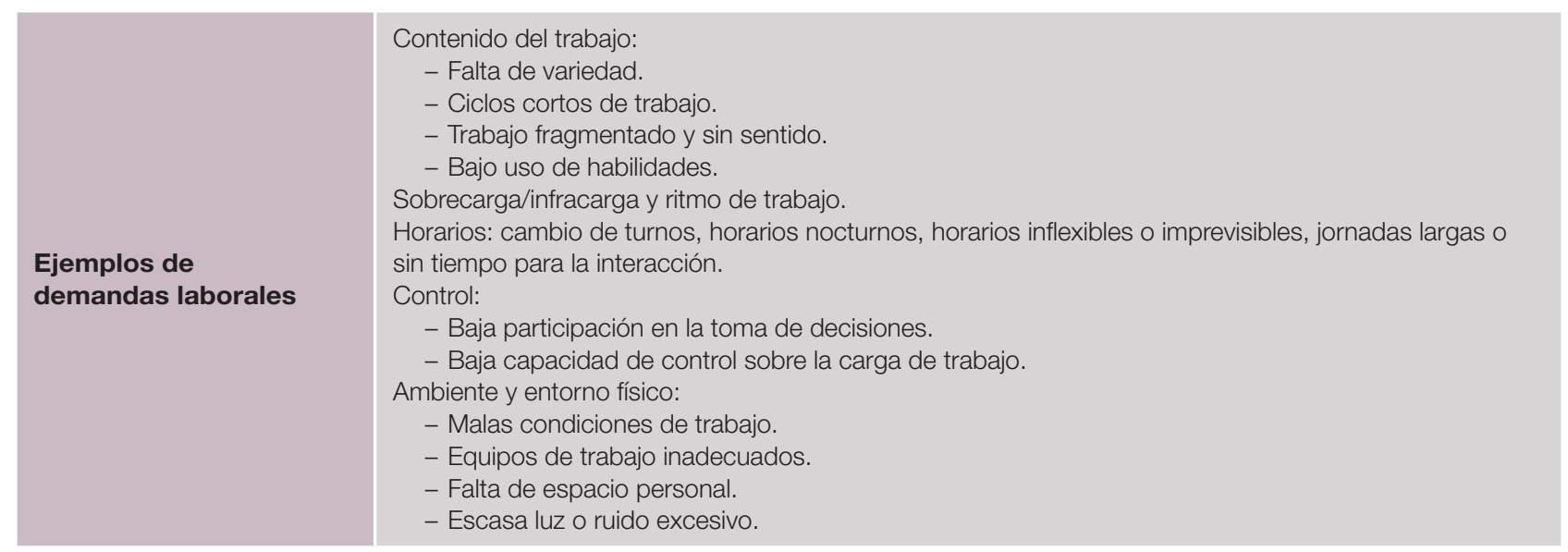

1. Hacemos aquí una presentación sintética del modelo de demandas-recursos (Demerouti y otros, 2001; Schaufeli y Bakker, 2004), que atribuye el bienestar de los trabajadores a las características de sus entornos de trabajo. 


\begin{tabular}{|c|c|}
\hline $\begin{array}{l}\text { Ejemplos de } \\
\text { demandas laborales }\end{array}$ & $\begin{array}{l}\text { Relaciones interpersonales: } \\
\text { - Aislamiento físico o social. } \\
\text { - Escasa relación con los jefes. } \\
\text { - Conflictos interpersonales. } \\
\text { - Falta de apoyo social. } \\
\text { Rol en la organización: } \\
\text { - Ambigüedad de rol: falta de información necesaria para desarrollar el trabajo. } \\
\text { - Conflicto de rol: situación en la que una persona recibe diferentes expectativas y demandas. } \\
\text { - Responsabilidad sobre otras personas. } \\
\text { Desarrollo de carrera incierto o inadecuado. } \\
\text { Conflicto trabajo-familia. }\end{array}$ \\
\hline $\begin{array}{l}\text { Ejemplos de } \\
\text { demandas laborales }\end{array}$ & $\begin{array}{l}\text { Inseguridad contractual. } \\
\text { Demandas emocionales: interacciones con carga emocional que se producen en el trabajo (por ejem- } \\
\text { plo, debido a un comportamiento inadecuado de clientes/colegas); la no posibilidad de mostrar las } \\
\text { propias emociones en el trabajo. }\end{array}$ \\
\hline $\begin{array}{l}\text { Ejemplos de recursos } \\
\text { laborales }\end{array}$ & $\begin{array}{l}\text { Sistema de recursos humanos: } \\
\text { - Retribución. } \\
\text { - Oportunidades de carrera. } \\
\text { - Seguridad del contrato. } \\
\text { Relaciones sociales: } \\
\text { - Apoyo del supervisor. } \\
\text { - Apoyo de los compañeros. } \\
\text { - Clima de equipo. } \\
\text { - Organización del trabajo: } \\
\text { - Claridad de rol: tener suficiente información sobre las responsabilidades y los objetivos del propio } \\
\text { puesto de trabajo dentro de la organización, y conocer qué comportamientos se consideran apro- } \\
\text { piados para alcanzar estos objetivos. } \\
\text { - Participación en la toma de decisiones. } \\
\text { Tarea: } \\
\text { - Variedad de destrezas necesarias. } \\
\text { - Identidad y significación de la tarea. } \\
\text { - Autonomía. } \\
\text { - Feedback sobre el rendimiento. }\end{array}$ \\
\hline $\begin{array}{l}\text { Ejemplos de recursos } \\
\text { personales }\end{array}$ & $\begin{array}{l}\text { Resiliencia: capacidad de una persona, cuando se enfrenta con la adversidad, para sobreponerse o } \\
\text { recuperarse de una contrariedad } \\
\text { o de un fracaso. } \\
\text { Autoeficacia: percepción sobre la propia capacidad para hacer frente } \\
\text { a las demandas en una amplia diversidad de contextos. } \\
\text { Optimismo: tendencia a creer que en general experimenta buenos resultados en la vida. } \\
\text { Esperanza: capacidad para planificar caminos hacia los objetivos deseados, a pesar de los obstáculos, } \\
\text { y la motivación para usar estos caminos. } \\
\text { Construcción de sentido: comprender por qué se ha producido un acontecimiento y cuál es su impacto. }\end{array}$ \\
\hline
\end{tabular}

Esta tabla ofrece ejemplos relevantes de cada uno de los elementos que pueden influir en el estrés de los trabajadores para facilitar la comprensión de estos conceptos. No se pretende ofrecer un listado exhaustivo de todas las demandas y recursos laborales y personales que se han estudiado hasta el momento en la literatura científica.

Fuente: Elaboración propia, a partir de Cox y Griffiths (1996), Demerouti y otros (2001), Schaufeli y Bakker (2004), Van den Heuvel y otros (2010).

Las investigaciones publicadas sugieren que, mientras que las demandas laborales normalmente son los principales predictores de resultados negativos para el bienestar, tales como el agotamiento o los problemas de salud psicosomáticos, los recursos son en general los predictores más importantes de resultados positivos como la satisfacción en el trabajo, la motivación y el engagement (vinculación psicológica con el trabajo).

Además, los recursos amortiguan el impacto de las demandas sobre el estrés/malestar. Diversos estudios han mostrado que recursos laborales tales como el apoyo social, la autonomía y la retroalimentación sobre el desempeño y las oportunidades de desarrollo pueden mitigar el impacto que tienen demandas laborales como la presión y las exigencias emocionales sobre varios indicadores de malestar. Es decir, que los trabajadores que disponen de muchos recursos pueden afrontar mejor sus demandas laborales diarias, en comparación con trabajadores con demandas similares pero menos recursos. 


\section{El impacto de los mandos sobre los recursos y las demandas laborales}

Cuando ya entendemos que demandas y recursos tienen impacto sobre el estrés, hay que preguntarse de qué o quién depende que un trabajador disponga de más recursos y haga frente a menos demandas. En buena medida (aunque en absoluto de forma exclusiva), esto depende de la actuación de los mandos. Un creciente número de autores ha sugerido que el comportamiento de los directivos -el liderazgo- tiene una importante influencia sobre el bienestar de los trabajadores (por ejemplo, Kelloway y Barling, 2010; Skakon y otros, 2010).

En cuanto a las demandas, es bastante evidente la influencia directa que los mandos pueden tener sobre ellas. Una reflexión cuidadosa sobre los principales riesgos psicosociales que afrontan los colaboradores puede ofrecer sugerencias de disminución de las demandas que están en manos de los directivos y pueden tener un coste insignificante en términos económicos, al tiempo que un gran beneficio en términos de bienestar. Tomando como ejemplo algunas de las demandas incluidas en la tabla 1, los mandos tienen la capacidad de asignar a sus colaboradores tareas diversas que requieran hacer un buen uso de sus habilidades, pedir su participación en la toma de decisiones que les afectan, o pueden aclarar posibles conflictos o ambigüedades de rol.

Pero, además de actuar sobre las características más objetivas del trabajo, los mandos pueden ejercer un impacto indirecto, influyendo sobre cómo interpreta el trabajador las demandas a las que hace frente. La investigación ha mostrado que no todas las demandas tienen igual impacto sobre el bienestar de los trabajadores, sino que depende de si estos las entienden como un «reto» o como un «obstáculo» (Crawford, Lépine y Rich, 2010). Los retos suelen ser valorados como demandas que pueden promover el dominio de una capacidad, el crecimiento personal y las ganancias futuras. Ejemplos de retos son demandas tales como una elevada carga de trabajo puntual y altos niveles de responsabilidad laboral. Los trabajadores tienden a percibir estas demandas como una oportunidad para aprender, alcanzar objetivos y demostrar el tipo de capacidades que suelen ser recompensadas. En contraste, los obstáculos normalmente son valorados como demandas estresantes, limitaciones o barreras que pueden llegar a impedir el crecimiento personal, el aprendizaje y el logro de metas. Son ejemplos de obstáculos el conflicto y la ambigüedad de rol, las intrigas y luchas de poder en la organización, la burocracia y las incomodidades físicas o tecnológicas.

Si los mandos tienen presente el potencial motivador de las demandas «reto», pueden orientarse a minimizar las demandas que realmente son «obstáculos» y, en la medida de lo posible, en transformar la visión de los trabajadores sobre algunas demandas que estos puedan entender como obstáculos. Por ejemplo, un directivo puede explicar cómo una nueva tarea no es solo una carga adicional para los trabajadores afectados, y mostrar cómo esta tarea mejora el potencial de crecimiento profesional de los trabajadores. También puede ayudar a comprender que una aparente contradicción entre directrices recibidas en realidad no es tal, o que cierto procedimiento burocrático que el trabajador debe cumplir está justificado y forma parte de la contribución del trabajador al éxito de la empresa.

Por otra parte, está en manos de los mandos aumentar los recursos a disposición de los trabajadores. Una lectura a la inversa de la primera fila de la tabla 1 ofrece claras sugerencias sobre cómo hacerlo. Entre los recursos que los mandos más fácilmente pueden favorecer están los relacionados con la tarea y la organización del trabajo, pero también pueden dar su apoyo social y facilitar que haya oportunidades de apoyo social entre colaboradores. Hay otros recursos que se han de implantar a nivel organizativo, como ofrecer contratos estables y flexibilidad en los horarios.

Pero si nos limitamos a decir que los directivos deben disminuir las demandas y aumentar los recursos, no estaríamos contando toda la historia. La investigación publicada sugiere además que unas elevadas demandas en el trabajo no parecen ser problemáticas si los trabajadores tienen suficientes recursos (Bakker, van Veldhoven y Xanthopoulou, 2010). De hecho, se ha encontrado que los trabajadores en trabajos con altas demandas y altos recursos son los que disfrutan más de la tarea y están más comprometidos con sus organizaciones. Por lo tanto, el simple hecho de disponer de muchos recursos no es suficiente para que aumente la vinculación positiva con el trabajo, sino que es necesario que haya un desafío (es decir, una situación exigente) para que estos recursos laborales se traduzcan en una superior implicación de la persona con el trabajo. Tener más recursos de los que las demandas requieren podría generar aburrimiento, un resultado claramente no deseable ni para los trabajadores ni para la empresa. 
Ahora bien, unas fuertes demandas, por desafiantes que sean y por muchos recursos que haya para hacerles frente, siempre generan cansancio. Es necesario ofrecer a los trabajadores oportunidades para recuperarse, para que puedan reponer energías y volver al trabajo sin arriesgar su salud o bienestar (Kinnunen y otros, 2011). Una de las principales formas como los mandos pueden facilitar esta recuperación es favoreciendo que los trabajadores se separen psicológicamente del trabajo en horario no laboral. Esto es más que irse físicamente del trabajo, ya que implica dejar de pensar sobre cuestiones laborales. Si los mandos tienen presente esta necesidad, evitarán -por ejemplo- las llamadas o los mensajes en horarios intempestivos, intentarán asegurarse de que las personas no se lleven trabajo a casa si ya han cumplido su jornada, procurarán que tras un periodo de alta presión de tiempo pueda haber al menos unos días con demandas más moderadas, etc.

\section{Los estilos de liderazgo y el bienestar psicosocial}

Las actuaciones que acabamos de comentar se materializarán en los comportamientos que desarrollan los directivos en su día a día, en lo que podemos llamar su estilo de liderazgo. Podemos definir el liderazgo como el proceso de influir en los miembros de un grupo de tal manera que motive a las personas a contribuir a que se alcancen los objetivos del grupo o la organización. Los estilos de liderazgo -el modo como los mandos tratan de influir sobre sus colaboradores- pueden clasificarse en una escala de más negativo a más positivo en cuanto a su impacto sobre el bienestar de los trabajadores: estilo abusivo en el extremo negativo, pasivo, transaccional y transformacional en el extremo positivo. Veamos en qué consiste cada uno de los estilos de liderazgo y de qué manera pueden influir sobre el bienestar de los trabajadores.

El liderazgo abusivo supone el uso sostenido de comportamientos hostiles, verbales y no verbales, excluyendo el contacto físico. Incluye, por ejemplo, poner en ridículo, culpar de errores no cometidos, intimidar, etc. Los estudios disponibles indican una relación del liderazgo abusivo con aspectos psicológicos como el burnout, la sensación de indefensión, la menor confianza en las propias capacidades, la autoestima y el compromiso con la organización. Por supuesto, hay otros estilos de liderazgo que, sin ser abusivos, podríamos calificar como «autocráticos» o dictatoriales que también empeoran la salud mental, la vitalidad y diversos comportamientos derivados del estrés (Nyberg y otros, 2011). Estas formas de liderazgo probablemente aumentan las demandas que deben afrontar los trabajadores, limitando al mismo tiempo los recursos con que estos cuentan para hacerles frente (libertad para decidir sobre las propias tareas, apoyo por parte del mando, feedback sobre el propio rendimiento, etc.), lo que necesariamente disminuirá su bienestar psicológico.

El estilo pasivo o laissez-faire supone un mando inactivo, que no está disponible cuando es necesario, no aclara las expectativas y evita la toma de decisiones. A pesar de ser pasivo, resulta destructivo, ya que incrementa demandas o estresores laborales como la ambigüedad de rol, el conflicto de rol y el conflicto con los colaboradores (Skogstad y otros, 2007).

En contraste con los dos estilos anteriores, el estilo de liderazgo transaccional supone que el mando comunica los objetivos a los trabajadores, supervisa activamente su evolución hacia estos objetivos y proporciona retroalimentación, recompensando el éxito o corrigiendo el desempeño deficiente. De este modo, disminuye ciertas demandas clave como el conflicto o la ambigüedad de rol, al tiempo que aumenta algunos recursos como el feedback sobre el propio rendimiento o las mejoras de carrera profesional o retributivas que se fijen como recompensa. En consecuencia, disminuye el burnout (Hetland y otros, 2007) y mejora la satisfacción de los trabajadores (Shieh y otros, 2001).

Por último, el liderazgo transformacional se orienta a transformar las normas y los valores de los trabajadores, de manera que el líder motiva a los trabajadores a obtener resultados más allá de sus propios intereses inmediatos, aumentando la implicación de los trabajadores con los objetivos de la organización. Esto se consigue a través de cuatro tipos de actuaciones: (a) inspiración motivadora, (b) influencia idealizada, (c) consideración individual y (d) estímulo intelectual (Bass y Avolio, 1990). La inspiración motivadora se muestra cuando el mando visualiza un futuro deseable y atractivo, y articula cómo se puede lograr. La influencia idealizada supone que el mando se comporta como ejemplo que seguir, establece altos estándares de rendimiento y muestra deter- 
minación y confianza. Los colaboradores suelen querer identificarse con un líder como este. La consideración individual se muestra cuando los mandos prestan atención a las necesidades de desarrollo de los colaboradores y les dan apoyo y asesoramiento en este sentido. Este líder delega responsabilidad como oportunidades para el crecimiento. Finalmente, el estímulo intelectual se muestra cuando el líder ayuda a los trabajadores a ser más innovadores y creativos (Bass, 1999). Cada uno de los cuatro componentes del liderazgo transformacional puede generar un impacto favorable sobre el bienestar y la salud de los colaboradores:

- La inspiración motivadora y el estímulo intelectual pretenden facilitar el cambio en los colaboradores y motivarlos a lograr más de lo que creían posible, haciendo que su trabajo tenga más sentido y proporcionándoles un sentido de propósito. Este tipo de comportamientos puede tener un impacto indirecto sobre el bienestar psicológico porque modifica el modo como los trabajadores experimentan su entorno laboral. Así, los trabajadores pueden interpretar más positivamente eventos potencialmente estresantes, y aumentar su satisfacción focalizándose en los aspectos agradables de alcanzar objetivos en vez de en los costes que implica.

- En segundo lugar, la consideración individual, la empatía y la preocupación de un mando por sus colaboradores pueden tener un impacto positivo directo sobre el nivel de estrés de estos. Además, hay un impacto indirecto de estos aspectos sobre el bienestar psicológico de los trabajadores, ya que mejoran recursos personales como el optimismo, la autoestima y la resiliencia, que a su vez contribuyen al bienestar físico y mental (Kelloway y otros, 2013). En definitiva, el apoyo de un mando directo es un recurso que puede hacer que las demandas no sean percibidas como una carga tan grande porque aumenta la capacidad de los trabajadores para hacerles frente.

- Por último, mediante la influencia idealizada el mando se convierte en un modelo que seguir para sus colaboradores. Por tanto, si pone en práctica las conductas positivas descritas a lo largo del artículo y, además, las aplica en su propia persona (por ejemplo, tomándose descansos para recobrar energía), es probable que este tipo de conductas también aumenten entre sus colaboradores. Además, puede mejorar el autoconcepto de los trabajadores porque mejora la percepción de confianza y de respeto que reciben del mando, el cual es considerado fiable y digno de admiración (Franke y Felfe, 2011).

Cabe destacar que cualquier mando presenta con cierta frecuencia todos o muchos de los estilos de liderazgo descritos. Los mandos que generan más satisfacción en sus colaboradores y que son más eficaces tienden a ser más transformacionales que transaccionales, aunque presenten en cierto grado ambos comportamientos (Bass, 1999). En este caso, las acciones del mando van fuertemente orientadas a disminuir las demandas de tipo "obstáculo", destacando los aspectos positivos de las de tipo «reto", y mejorando los recursos laborales -y en cierta medida también los personales- de los trabajadores.

En conjunto, pues, podemos decir que los estilos de liderazgo transaccional y transformacional tienen un elevado potencial de impacto favorable sobre varias causas de estrés y otros indicadores de bienestar psicosocial como la satisfacción del trabajador. Diversos estudios así lo han demostrado, entre los que destaca uno bastante reciente que agrupaba datos referentes a 93.576 trabajadores en 11.117 equipos de una gran empresa multinacional. En él se encontraron claras evidencias del efecto promotor de la salud del liderazgo transformacional y de las recompensas contingentes (un componente clave del liderazgo transaccional), así como del efecto perjudicial para la salud del liderazgo pasivo (Zwingmann y otros, 2014). Es, por tanto, deseable promover los estilos de liderazgo más positivos entre los mandos de nuestras empresas y organizaciones.

\section{Conclusión}

A lo largo del artículo hemos explicado que el bienestar psicológico de los trabajadores deriva de un adecuado equilibrio entre, por un lado, los retos motivadores y los obstáculos a los que hacen frente y, por otro, los recursos laborales y personales con que cuentan para superarlos. También hemos explicado cómo los directivos o mandos están en una posición privilegiada para hacer posible este equilibrio, a través de su comportamiento diario. En 
concreto, los estilos de liderazgo transaccional y, sobre todo, transformacional pueden impactar favorablemente sobre diversos factores de riesgo psicosocial, disminuyendo de paso el estrés de los trabajadores.

Si liderar de una manera transformacional contribuye al bienestar de los trabajadores, además de a la eficacia de la organización, es importante promover la formación en liderazgo entre nuestros mandos. No parece demasiado difícil aprender a ser un mejor líder transaccional (fijar objetivos y recompensas, hacer el seguimiento, corregir constructivamente, etc.). Más complejo es desarrollar la capacidad de ser un líder transformacional. Sin embargo, es posible. Hay estudios que demuestran la eficacia de las acciones formativas orientadas en este sentido (véase Kelloway y Barling, 2010). Pero para que esta formación tenga lugar y sea exitosa hay dos factores clave que no podemos dar por hechos. Primero, es necesario o bien que las empresas y organizaciones pidan a sus mandos que desarrollen y pongan en práctica estas conductas, o bien que los propios mandos se formen motu proprio en liderazgo transformacional. Segundo, es necesario que las instituciones responsables de formar directivos estén a la altura y vayan más allá de la formación técnica, reforzando también las competencias de tipo social y ético que caracterizan a los líderes transformacionales.

En conclusión, si queremos menos estrés en el trabajo, hay que potenciar una «alfabetización» en materia de liderazgo que implique a los mandos, las empresas y organizaciones y, también, pedir a las entidades formadoras que apliquen metodologías pedagógicas contrastadas para desarrollar estilos de liderazgo transformacionales.

\section{Bibliografía}

BAKKER, A. B.; VAN VELDHOVEN, M.; XANTHOPOULOU, D. (2010). «Beyond the Demand-Control model: Thriving on high job demands and resources». Journal of Personnel Psychology. Vol. 9, núm. 1, pág. 3-16.

BASS, B. M.; AVOLIO, B. J. (1990). «Developing transformational leadership: 1992 and beyond». Journal of European Industrial Training. Vol. 14, núm. 5.

BASS, B. M. (1999). «Two decades of research and development in transformational leadership». European Journal of Work and Organizational Psychology. Vol. 8, núm. 1, pág. 9-32.

COX, T.; GRIFFITHS, A. J. (1996). «The assessment of psychosocial hazards at work». En: SCHABRACQ, M. J.; WINNUBST, J. A. M.; COOPER, C. L. (eds.). Handbook of Work and Health Psychology. Chichester: Wiley and Sons, pág. 127-146

CRAWFORD, E. R.; LEPINE, J. A.; RICH, B. L. (2010). «Linking job demands and resources to employee engagement and burnout: a theoretical extension and meta-analytic test». Journal of Applied Psychology. Vol. 95, núm. 5, pág. 834.

DEMEROUTI, E.; BAKKER, A. B.; NACHREINER, F.; SCHAUFELI, W. B. (2001). «The job demands-resources model of burnout». Journal of Applied Psychology. Núm. 86, pág. 499-512.

HETLAND, H.; SANDAL, G. M.; JOHNSEN, T. B. (2007). «Burnout in the information technology sector: Does leadership matter?». European Journal of Work and Organizational Psychology, núm. 16, pág. 58-75.

FRANKE, F.; FELFE, J. (2011). «How does transformational leadership impact employees' psychological strain? Examining differentiated effects and the moderating role of affective organizational commitment». Leadership. Vol. 7, núm. 3, pág. 295-316.

KELLOWAY, E. K.; BARLING, J. (2010). «Leadership development as an intervention in occupational health psychology». Work \& Stress. Vol. 24, núm. 3, pág. 260-279.

KELLOWAY, E. K.; WIGAND, H.; MCKEE, M. C.; DAS, H. (2013). «Positive Leadership and Employee Well-Being». Journal of Leadership \& Organizational Studies. Vol. 20, núm. 1, pág. 107-117.

KINNUNEN, U.; FELDT, T.; SILTALOPPI, M.; SONNENTAG, S. (2011). "Job demands-resources model in the context of recovery: Testing recovery experiences as mediators". European Journal of Work and Organizational Psychology. Vol. 20, núm. 6, pág. 805-832.

LEVI, L.; LEVI, I. (2000). Guidance on work-related stress - Spice of life or kiss of death? Luxemburgo: Office for Official Publications of the European Communities. 
NYBERG, A.; HOLMBERG, I.; BERNIN, P.; ALDERLING, M. (2011). «Destructive managerial leadership and psychological well-being among employees in Swedish, Polish, and Italian hotels». Work: A Journal of Prevention, Assessment and Rehabilitation. Vol. 39, núm. 3, pág. 267-281.

SCHAUFELI, W. B.; BAKKER, A. B. (2004). «Job demands, job resources, and their relationship with burnout and engagement: A multi-sample study». Journal of Organizational Behavior. Núm. 25, pág. 293-315.

SHIEH, H.-L.; MILLS, M. E.; WALTZ, C. E. (2001). «Academic leadership style predictors for nursing faculty job satisfaction in Taiwan». Journal of Nursing Education. Núm. 40, pág. 203-209.

SKAKON, J.; NIELSEN, K.; BORG, V.; GUZMAN, J. (2010). «Are leaders' well-being, behaviours and style associated with the affective well-being of their employees? A systematic review of three decades of research». Work \& Stress. Vol. 24, núm. 2, pág. 107-139.

SKOGSTAD, A.; EINARSEN, S.; TORSHEIM, T.; AASLAND, M. S.; HETLAND, H. (2007). «The destructiveness of laissez-faire leadership behavior». Journal of Occupational Health Psychology. Vol. 12, núm. 1, pág. 80.

VAN DEN HEUVEL, M.; DEMEROUTI, E.; SCHAUFELI, W. B.; BAKKER, B. (2010). «Personal resources and work engagement in the face of change». En: HOUDMONT, J.; LEKA, S. (eds.). Contemporary Occupational Health Psychology: Global perspectives on research, education, and practice. Chichester, England: Wiley-Blackwell, vol. 1, pág. 124-150.

XANTHOPOULOU, D.; BAKKER, B.; DEMEROUTI, E.; SCHAUFELI, W. B. (2007). «The Role of Personal Resources in the Job Demands-Resources Model». International Journal of Stress Management. Vol. 14, núm. 2, pág. 121-141.

ZWINGMANN, I.; WEGGE, J.; WOLF, S.; RUDOLF, M.; SCHMIDT, M.; RICHTER, P. (2014). «IS transformational leadership healthy for employees? A multilevel analysis in 16 nations». Zeitschrift fuer Personalforschung. German Journal of Research in Human Resource Management. Vol. 28, núm. 1-2, pág. 24-51.

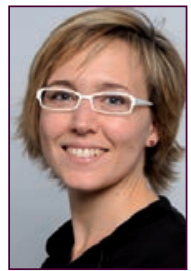

\section{Fva Rimbau-Gilabert} erimbau@uoc.edu Profesora de la Universitat Oberta de Catalunya (UOC). Estudios de Economía y Empresa

Profesora en la UOC desde el año 2004, donde ha desarrollado el cargo de directora académica de la licenciatura de Ciencias del Trabajo, del máster universitario de Prevención de riesgos laborales, y del Área de Posgrado de Economía y Empresa. Es miembro del grupo de investigación consolidado DigiBiz (Digital Business Research Group), del IN3-Universitat Oberta de Catalunya. Ha sido investigadora residente del IN3, donde desarrolló el proyecto «Flexible work arrangements and business performance». Sus intereses de investigación se centran en las formas de trabajo flexibles, el trabajo virtual y la dirección de personas en organizaciones intensivas en conocimiento, con un interés especial sobre el rol de los directivos en estos entornos.

Los textos publicados en esta revista están -si no se indica lo contrario- bajo una licencia Reconocimiento-Sin obras derivadas 3.0 España de Creative Commons. Puede copiarlos, distribuirlos y comunicarlos públicamente siempre que cite su autor y la revista y la institución que los publica (autoría, nombre de la revista, institución editora); no haga con ellos obras derivadas. La licencia completa se puede consultar en http://creativecommons.org/licenses/by-nd/3.0/es/deed.es.

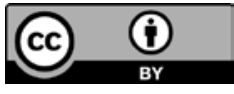

